Revue d'histoire du XIXe siècle

Société d'histoire de la révolution de 1848 et des

révolutions du XIXe siècle

$52 \mid 2016$

Chrononymes. Dénommer le siècle

\title{
Robert J. MAYHEW, Malthus, The Life and Legacies of an Untimely Prophet
}

Cambridge (Mass.), Harvard University Press, 2014

Patrice Bouche

\section{(2) OpenEdition \\ Journals}

Édition électronique

URL : http://journals.openedition.org/rh19/5011

DOI : 10.4000/rh19.5011

ISSN : $1777-5329$

Éditeur

La Société de 1848

Édition imprimée

Date de publication : 1 juin 2016

Pagination : 200-202

ISSN : 1265-1354

Référence électronique

Patrice Bouche, «Robert J. MAYHEW, Malthus, The Life and Legacies of an Untimely Prophet », Revue d'histoire du XIXe siècle [En ligne], 52 | 2016, mis en ligne le 01 juin 2016, consulté le 23 septembre 2020. URL : http://journals.openedition.org/rh19/5011; DOI : https://doi.org/10.4000/rh19.5011

Ce document a été généré automatiquement le 23 septembre 2020.

Tous droits réservés 


\section{Robert J. MAYHEW, Malthus, The Life and Legacies of an Untimely Prophet}

Cambridge (Mass.), Harvard University Press, 2014

Patrice Bouche

\section{RÉFÉRENCE}

Robert J. MAYHEW, Malthus, The Life and Legacies of an Untimely Prophet, Cambridge (Mass.), Harvard University Press, 2014, 284 p., 28,50 euros.

1 Ce nouvel ouvrage sur Malthus n'est pas réellement une biographie (contrairement au Population Malthus ${ }^{1}$ de Patricia James, qui suivait pas à pas sa "carrière»), ni un commentaire systématique de ses écrits (proposé en 2013, sous forme condensée, par Donald Winch'). À l'instar de bien d'autres « défenseurs » de Malthus, Robert Mayhew s'attache à nous rendre le « Pasteur » aimable. Mais, contrairement à Patricia James, il considère que la vie de Malthus fut trop ordinaire pour la narrer en détail. Seul l'impact durable de son premier Essai sur le principe de population fit de lui un auteur hors pair, comme le suggèrent les effets d'annonce dont l'auteur use pour dramatiser le récit de la composition originelle de l'Essai («Where did Malthus's unique voice come from, why did that voice come to find its muse in the events of $1798 \ldots$ ? Those are the questions we will address in this chapter. », p. 53). Mayhew fait par ailleurs de la banalité même de la vie de Malthus un ressort quasi-comique (par exemple, en mentionnant les préoccupations très familiales du jeune pasteur à l'été 1789). En outre, le fait que, selon lui, le récit biographique ne puisse réellement démarrer, faute d'éléments pour le nourrir, donne lieu à un rapprochement tout littéraire avec le Tristram Shandy de Sterne. Cette référence insolite peut apparaitre comme un procédé pour nous rendre Malthus sympathique. Mais un autre parallèle littéraire avec Jane Austen, dont la vie privée fut bien plus ordinaire que celle de Malthus, permet de comprendre que le but de Mayhew est de montrer que le style peut transcender le vécu d'un auteur : point n'est besoin de vivre directement les événements majeurs de son siècle pour en tirer 
des réflexions pénétrantes. En s'attachant à retrouver ce que l'on peut appeler le "grain de la vie » à l'époque où fut écrit l'Essai, au travers de journaux intimes de contemporains, Mayhew veut nous donner à ressentir la précarité de l'existence telle qu'elle se présentait à Malthus. On peut voir dans cette volonté de rassembler le matériau qui constitua "Malthus's unique voice» une approche déterministe. Outre le vécu sans relief de l'économiste, qui était en 1798 un célibataire trentenaire vivant encore aux crochets de son père (ce qui l'aurait rendu naturellement conscient de la nécessité de pratiquer le "preventive check»), Mayhew dresse la liste des outils intellectuels de Malthus : la pensée malthusienne serait le fruit d'une alliance singulière entre la vision newtonienne d'un univers régi par des lois immuables, et un esprit satirique, certes moins acéré que celui de Swift, mais que le lecteur averti détectera de façon répétée dans l'Essai.

2 Les écrits de Malthus ne sont néanmoins que peu cités et analysés très brièvement. En outre, si Mayhew évoque les contributions de Malthus à la théorie économique, notamment la notion de "demande effective", il le fait sans technicité, et dans le simple but de montrer l'originalité de sa pensée. Mayhew déplore certes, comme tous ses apologistes, que Malthus soit, depuis deux siècles, bien davantage critiqué que réellement lu. Mais cet ouvrage est précisément destiné à un public qui aura lu Malthus. La "réhabilitation» du sens et de la portée des écrits de Malthus est, selon Mayhew, toujours à recommencer. Mais la raison en est que le discours anti-malthusien renaît perpétuellement, au gré des oscillations économiques. C'est là pour l'auteur la preuve insigne $\mathrm{du}$ caractère incontournable de Malthus. Historiquement, cette dialectique aurait débuté avec les poètes romantiques (notamment Coleridge, Southey, Byron, Shelley), qui, tout en contribuant largement à façonner l'image sinistre de Malthus, lui devraient en grande partie la définition de leur vision (" rédemptrice ») de la nature. C'est d'ailleurs en opposant Malthus à Southey (qui, contrairement à l'économiste, voyait dans l'impérialisme la solution aux problèmes démographiques britanniques), mais aussi, et par ailleurs, à Burke (qui mettait la grande population française au crédit de l'Ancien régime), que Mayhew bat en brèche sa réputation de chantre du conservatisme.

3 Contrairement à deux autres publications de 2014, l'objet d'analyse de ce Malthus n'est ni l'impact de la pensée malthusienne sur tel ou tel domaine (traité par Piers Hale concernant le darwinisme ${ }^{3}$ ), ni la nature même de cette pensée (examinée dans son rapport à l'utilitarisme benthamien par Sergio Cremaschi $\left.{ }^{4}\right)$. Mayhew veut essentiellement démontrer la résilience du Principe de population, en passant en revue les voies diverses empruntées par la controverse malthusienne. À cette aune, Carlyle et Ruskin peuvent côtoyer Engels et Marx, mais aussi H.G. Wells et Aldous et Julian Huxley, puis Paul Ehrlich et Julian Simon, pour aboutir à Al Gore. En effet, dans la deuxième moitié de l'ouvrage, qui s'ouvre symboliquement sur une évocation de la mort de Malthus, Mayhew aligne une véritable galerie d'auteurs, au rythme soutenu d'une à deux pages dévolues à chacun d'entre eux, à l'exception de développements un peu plus longs pour Darwin/Wallace et surtout pour Keynes, qui ont contribué à « réinventer » Malthus, ou du moins à en définir notre perception actuelle.

4 Mayhew dit vouloir résister à la tentation d'attribuer à Malthus des idées environnementales propres à notre époque. Un glissement s'opère néanmoins dans la nature de la controverse, telle qu'illustrée par les auteurs susmentionnés, de la question sociale originelle à l'impact de l'activité humaine sur les ressources naturelles, 
débat inauguré dès le XIX ${ }^{e}$ siècle par Jevons et Marshall. Toutefois, à qui refuse ce rapprochement de la pensée malthusienne avec la conscience écologique moderne, on aura beau jeu de répliquer que les anti-malthusiens actuels ont moins de prévention encore à attribuer à Malthus la paternité des pires crimes du XX $\mathrm{XX}^{\mathrm{e}}$ siècle. Ainsi, l'auteur ne craint pas d'affronter ce que l'on nomme aujourd'hui le «point Godwin » de tout débat (nous parlons ici de l'homonyme moderne de William Godwin!), en évoquant le lien, fort indirect mais indéniable, entre le Principe de population et la requalification par les Nazis des populations «non-aryennes » en populations surnuméraires (p. 181). Mayhew désamorce toutefois ce rapprochement d'une façon assez audacieuse, en montrant que l'Empire britannique usa d'un langage analogue en Inde, notamment par la plume du jeune Churchill lui-même. Enfin, face aux arguments les plus gênants contre Malthus, à commencer par son infamante parabole du "Banquet de la Nature " (auquel, selon la deuxième édition de l'Essai, l'individu que ses parents ne peuvent nourrir n'a aucun droit de participer), l'auteur oppose à la portée « théorique » de cette parabole d'autres propos bien plus humains de l'économiste sur la nécessité d'atténuer la disette des années de guerre contre la France. Pourtant, comme on l'a vu, l'argument principal de cet ouvrage, attrayant dans son style et son approche, est, non pas tant de protester de l'humanité de Malthus, que de s'émerveiller qu'un homme, écrivant à contre-courant de son temps, ait pu occasionner des débats qui se perpétuent en son nom depuis deux siècles.

\section{NOTES}

1. P. James, Population Malthus: His Life and Times, Londres, Routledge, 1979

2. D. Winch, Malthus, a Very Short Introduction, Oxford, Oxford University Press, 2013

3. Piers J. Hale, Political Descent : Malthus, Mutualism and the Politics of Evolution in Victorian England, University of Chicago Press, 2014.

4. Sergio Cremaschi, Utilitarianism and Malthus' Virtue Ethics: Respectable, Virtuous and Happy, Routledge, 2014. 